\title{
Toxico-Surveillance of Infant and Toddler Poisonings in the United States
}

\author{
Yaron Finkelstein • Janine R. Hutson • Paul M. Wax • \\ Jeffrey Brent • \\ on behalf of the Toxicology Investigators Consortium \\ (ToxIC) Case Registry
}

Published online: 20 April 2012

(C) American College of Medical Toxicology 2012

\begin{abstract}
Infant and toddler poisonings are important to capture and may be challenging to manage. We aim to describe the Toxicology Investigators Consortium (ToxIC) Case Registry as a tool for toxico-surveillance of this problem in the United States. Using the ToxIC Case Registry database of the American College of Medical Toxicology, we identified infant and toddler poisonings over a 15-month period between April 1, 2010 and June 30, 2011 reported to the 31 Registry sites. Of 6,810 poisoning cases reported to
\end{abstract}

\section{Y. Finkelstein}

Division of Emergency Medicine, Department of Pediatrics,

Hospital for Sick Children, University of Toronto,

Toronto, ON, Canada

\section{Y. Finkelstein · J. R. Hutson}

Division of Clinical Pharmacology,

Clinical Pharmacology and Toxicology, Department of Pediatrics,

Hospital for Sick Children, University of Toronto,

Toronto, ON, Canada

\section{Y. Finkelstein}

Clinical Pharmacology Research Program,

Division of Emergency Medicine, Children's Hospital Boston,

Boston, MA, USA

P. M. Wax

University of Texas Southwestern Medical School,

Dallas, TX, USA

\section{J. Brent}

Toxicology Associates,

School of Medicine and Colorado School of Public Health, University of Colorado,

Denver, CO, USA

Y. Finkelstein $(\triangle)$

Divisions of Emergency Medicine and Clinical Pharmacology and Toxicology, Hospital for Sick Children, University of Toronto, 555 University Ave,

Toronto, ON M5G 1X8, Canada

e-mail: Yaron.Finkelstein@sickkids.ca the ToxIC registry, 248 (3.6\%) involved children younger than 2 years (51\% males). Fifty-four percent were hospital inpatients, $42 \%$ were in the Emergency Department and $4 \%$ were outpatients. Sixty-three percent were symptomatic. The most common ingested compounds were highly toxic-cardiac drugs (16\%), psychotropics (15\%), recreational drugs, alcohols, and controlled narcotic drugs (13\%), analgesics (9\%), and cleaning compounds $(7 \%)$. Fourteen percent of cases involved multiple agents. The ToxIC registry is a potentially useful toxico-surveillance tool to identify and trend clinically significant poisonings in young children, and potentially other populations. These data could be used to target specific preventive interventions.

Keywords Poisoning $\cdot$ Infants $\cdot$ Toxicology $\cdot$ Case registry

\section{Introduction}

Infant and toddler poisonings are a particularly important public health concern, since toxicological adverse effects in this age group have greater potential ramifications in terms of person years-of-life than poisonings in adolescents and adults [1]. In 2009, the American Association of Poison Control Centers (AAPCC) reported over 1.6 million pediatric poison exposures and the incidence is increasing annually [2]. Of these, 534,236 (33.1\%) occurred in children younger than 2 years and approximately $90 \%$ of them were managed at home. In the United States, 41,375 infants and toddlers visited emergency departments (ED) in 2004 for poisoning, corresponding to a poisoning rate of 2.2 and 8.0 per 1,000 children aged $<1$ year and 1 to 2 years, respectively [1]. Visits to the ED in children $\leq 5$ years of age increased by $30 \%$ between 2001 and 2008 in the USA and poisoning prevention efforts to date are inadequate [3]. 
Although estimates of possible pediatric poisonings of varying severity are reported in the above citations, there are sparse data about exposure patterns of confirmed consequential and serious poisonings in this age group. Data from the poison control centers (PCCs) suggest that the most common agents involved in these exposures are household cleaning products and analgesics, and the vast majority of which are managed without referral to a medical facility [2]. Thus, further observational data are required to identify which agents are responsible for the more serious exposures, resultant ED visits and hospitalizations in order to make progress in reducing childhood injury from poisoning [3].

We sought to analyze the poisonings in children less than 2 years old reported to the Toxicology Investigators Consortium (ToxIC) Case Registry over a 15 -month period, in order to identify substances responsible for consequential exposures in this age group across the United States (US).

\section{Methods}

In 2010, the American College of Medical Toxicology (ACMT) established a prospective, nationwide toxicology database, The ToxIC Case Registry [4]. This registry is exclusively compiling cases that have been consulted and managed by a medical toxicologist at the bedside in 31 participating medical centers that have established medical toxicology consultation programs across the US. Using prospectively collected data from the ToxIC Case Registry, we identified all cases of poisoning in children up to 2 years, who have been consulted on at the bedside by a medical toxicologist between April 1, 2010 and June 30, 2011. Most of these sites are academic institutions, which collectively constitute the majority of medical toxicology services in the US. Participating sites prospectively enroll and report all patients that are cared for by the medical toxicology service at each center.

Detailed demographic and clinical data are collected on each patient including, but not restricted to, age group, location of initial encounter with the patient, circumstances and reasons for exposure, substances involved, presence of clinical signs and symptoms, and treatments provided. Lack of an identified toxin served as an exclusion criteria. Sites contribute cases to the ToxIC Registry pursuant to approval by their Institutional Review Boards.

\section{Results}

Of 6,810 poisoning cases reported to ToxIC registry during the 15 -month study period, 248 (3.6\%) involved children less than 2 years. Of them, the toxin was identified in 223 children $(90 \%)$, who form the study cohort. Gender was reported in 216 (110 were males; $51 \%$ ). Location of initial encounter was reported in 195 cases: 105 (54\%) were consulted while admitted to hospital, 82 (42\%) were consulted while still in the Emergency Department and 8 (4\%) had an outpatient consult. One hundred forty $(63 \%)$ children exhibited clinical signs and/or symptoms at the time of consultation.

Table 1 summarizes the top seven exposure classes in our patient cohort. The most common groups of exposures were cardiovascular drugs $(n=36 ; 16 \%)$, psychotropic drugs $(n=34 ; 15 \%$, mostly antipsychotics followed by antidepressants and anticonvulsants), recreational and controlled narcotic drugs ( $n=20 ; 9 \%$, mostly methadone), analgesics ( $n=19 ; 9 \%)$, cleaning products $(n=16 ; 7 \%)$, scorpion stings $(n=11 ; 5 \%)$, and toxic alcohols $(n=9 ; 4 \%)$. Consultation for iron poisoning was requested for a single case only. Fourteen percent $(n=32)$ of the children had histories of ingestion of multiple agents.

\section{Discussion}

Poisoning in the first 2 years of life makes up $4 \%$ of all cases consulted at the bedside by medical toxicologists participating in the ToxIC registry. Our data demonstrate that, contrary to expectations based from AAPCC data, cardiovascular and psychotropic medications were the drug classes responsible for a significant proportion of poisonings.

The capturing of infant and toddler poisoning cases is especially important for several reasons. These children are vulnerable and helpless in protecting themselves from accidental or intentional environmental hazards, as well as communicating the events leading to injury. About $13 \%$ of our patients were exposed to recreational drugs, alcohols, or

Table 1 Most frequent infant exposures reported to the ToxIC Registry between April 1, 2010 and June 30, 2011 and the corresponding pediatric exposure rates reported to the American Association of Poison Control Centers (AAPCC) in 2009 [2]

\begin{tabular}{lll}
\hline Agent & $\begin{array}{l}\text { Percentage of cases } \\
\text { from ToxIC registry }\end{array}$ & $\begin{array}{l}\text { Percentage of cases } \\
\text { reported to AAPCC }\end{array}$ \\
\hline Cardiac drugs & $16 \%$ & $1.9 \%$ \\
Psychotropic drugs & $15 \%$ & $2.3 \%$ \\
Recreational and & $9 \%$ & $\mathrm{NR}$ \\
$\begin{array}{l}\text { controlled narcotic } \\
\text { drugs }\end{array}$ & \\
Analgesics & $9 \%$ & $9.7 \%$ \\
Cleaning products & $7 \%$ & $9.3 \%$ \\
Scorpion stings & $5 \%$ & $\mathrm{NR}$ \\
Toxic alcohols & $4 \%$ & $2.0 \%$ \\
\hline
\end{tabular}

$N R$, not reported since not in the top 25 agents

${ }^{\text {a }}$ For those $<2$ years of age

${ }^{\mathrm{b}}$ For those $\leq 5$ years of age 
controlled narcotic drugs. These data raise the concern of neglect, unsafe environment and child maltreatment. This is most relevant in first-year-of-life exposures, where purposeful motor and coordination skills required to access drugs are largely limited. In malicious pediatric poisonings, an age of less than 2 years is associated with life threatening symptoms, residual disability, and increased risk of death [5]. Such cases are unlikely to be voluntarily reported to PCCs by caregivers, and clinicians should have high index of suspicion for nonaccidental poisonings when confronted with these scenarios. Thus, toxico-surveillance systems, such as the ToxIC registry, can play a potential role in identifying these cases as well as other exposure trends. Similar to our findings, a recent study suggested that the majority $(>80 \%)$ of infant poisonings that attended the ED were classified as "toxic", having a significant potential for an adverse effect. Only a small proportion (19\%) of exposure cases presenting to the ED was reported to the regional PCC [6].

At the same time, capturing infant poisonings may be challenging. Epidemiological estimates from both the AAPCC data and from nationwide ED records via the National Electronic Injury Surveillance System-All Injury Program (NEISS-AIPO) are both believed to significantly underestimate the true magnitude of the problem [7-9]. In that respect, the ToxIC and AAPCC registries complement each other, providing an effective, real-time toxicosurveillance coverage in the United States. However, they differ in several aspects. First, AAPCC data rely on voluntary self-reporting. In children less than 5 years of age, there is an estimated sevenfold under-reporting of poison-related fatalities to PCCs and an even higher under-estimation of non-fatal cases [5]. Guardians of poisoned infants that attend the ED are not likely to have previously contacted a PCC [6-10]. The ToxIC case registry addresses that gap by documenting all inadvertent exposure presenting to participating sites and requiring bedside consultation.

Second, there are major differences between the type of exposures reported to AAPCC and those captured by the ToxIC Case Registry (Table 1). In the APPCC database, the most common possible exposures for children younger than 5 years are to household items, such as cosmetics and personal care $(13 \%)$, cleaning products $(9.3 \%)$, and foreign bodies/toys ( $7 \%$ ), and the majority were managed at home without referral to a healthcare facility [2]. The most common category of pharmaceutical exposure was analgesics $(9.7 \%)$ and only a small percentage was related to prescription psychotropic medications (2.3\%; Table 1). Many of the AAPCC cases are reported as "possible" exposures, where ingestions were not witnessed by the infant's guardian and have no analytical confirmation, and about $90 \%$ are perceived mild enough to be managed at home [2]. In contrast, ToxIC registry patients typically represent serious exposures, where front-line clinicians feel the greatest concern and request toxicology expertise. The ToxIC data show a profile of poisonings dominated by classes of medications and compounds associated with potentially high morbidity. Our findings are corroborated by a recent study in children younger than 6 years, which investigated exposures to a single pharmaceutical agent. The authors found that exposure to prescription medications is on the rise, and concluded that prevention efforts to date are inadequate [3].

In recent years, awareness to the risks of poison or chemical exposure and bioterrorism has increased, and a number of highly publicized terrorist events have heightened community fears. This has led to the establishment of several mechanisms for population surveillance outside the US, such as the Alerting and Surveillance Using Poisons Information Systems [11] as well as other medical informatics platforms [12]. Recent attempts to explore patterns and magnitude of consequential pediatric poisonings have also been made outside North America: the Spanish Society of Paediatric Emergencies reported 91 poisonings in children younger than 5 years over 1 year through their Toxicology Surveillance System-Intoxications Working Group [13]. Of the 91 cases, $70 \%$ were exposed to acetaminophen or cough and cold medications. Another recent retrospective study from Istanbul, Turkey reported on 330 poisoning hospitalizations in children up to 14 years [14]. The majority of exposures were to pharmaceutical agents (76\%). Of the 330 cases, $29(9 \%)$ were below 1 year of age, and all the latter were considered accidental. Of the 28 infants that had available data, 16 were exposed to pharmaceutical agents.

The main limitation of the study stems from the fact that the ToxIC registry is a sentinel system in mostly academic tertiary care institutions and, therefore, may not be representative of the experience and practice in primary healthcare facilities. However, its strength is that it is intended to describe confirmed, clinically significant and important poisonings. In summary, using a novel toxico-surveillance tool, we identified substances that produce significant poisoning morbidity and mortality in infants and toddlers. The oftencited established toxico-surveillance [2] system describes potential rather than actual poisonings. These findings raise, once again, the issue of infant access to medications and the need to establish a safer environment, both on the personal level (e.g., open containers left at hand-reach) as well as the role of industry and regulatory agencies in ensuring patient safety by targeted efforts (e.g., child-resistant packaging). There may be a need for specific interventions targeted at the high-risk medications, for example, blister or other types of unit dose packaging. Clinicians should maintain high index of suspicion to the possibility of unusual exposures in infants and toddlers, such as illicit drugs and alcohol. 
Financial Disclosure/Conflict of Interest The authors reported no financial disclosures relevant to this article.

\section{References}

1. Franklin RL, Rodgers GB (2008) Unintentional child poisonings treated in United States hospital emergency departments: national estimates of incident cases, population-based poisoning rates, and product involvement. Pediatrics 122(6):1244-1251

2. Bronstein AC, Spyker DA, Cantilena LRJ, Green JL, Rumack BH, Giffin SL (2009) Annual Report of the American Association of Poison Control Centers' National Poison Data System (NPDS): 27th Annual Report. Clin Toxicol (Phila) 48(10):979-1178

3. Bond GR, Woodward RW, Ho M (2012) The growing impact of pediatric pharmaceutical poisoning. J Pediatr 160(2):265-270.el

4. Wax PM, Kleinschmidt KC, Brent J (2011) The Toxicology Investigators Consortium (ToxIC) Registry. J Med Toxicol 7(4):259265

5. Yin S (2010) Malicious use of pharmaceuticals in children. J Pediatr 157(5):832-836

6. Polivka BJ, Casavant M, Baker SD (2010) Factors associated with healthcare visits by young children for nontoxic poisoning exposures. J Community Health 35(6):572-578
7. Setlik J, Ho M, Bond GR (2010) Emergency department use after pediatric pharmaceutical ingestion: comparison of two national databases. Clin Toxicol (Phila) 48(1):64-67

8. Blanc PD, Jones MR, Olson KR (1993) Surveillance of poisoning and drug overdose through hospital discharge coding, poison control center reporting, and the Drug Abuse Warning Network. Am J Emerg Med 11(1):14-19

9. Blanc PD, Kearney TE, Olson KR (1995) Underreporting of fatal cases to a regional poison control center. West J Med 162(6):505509

10. Chafee-Bahamon C, Lovejoy FHJ (1983) Effectiveness of a regional poison center in reducing excess emergency room visits for children's poisonings. Pediatrics 72(2):164-169

11. Waring WS, Palmer SR, Bateman DN (2007) Alerting and Surveillance Using Poisons Information Systems (ASPIS): outcomes from an international working group. Clin Toxicol 45:543-548

12. Watts M, Fountain J, Reith DM, Herbison P (2003) Clinical utility of an electronic poisons information and clinical decision support tool. Int J Med Inform 71:3-8

13. Azkunaga B, Mintegi S, Bizkarra I, Fernandez J (2011) Toxicology surveillance system of the Spanish Society of Paediatric Emergencies: first-year analysis. Eur J Emerg Med 18(5):285-287

14. Akin Y, Agzikuru T, Comert S, Atilkan P, Erdag GC, Telatar B (2011) Hospitalizations for pediatric intoxication: a study from Istanbul. Turk J Pediatr 53(4):369-374 